\title{
Title: VIL1, a Polycomb-associated protein, modulates high ambient temperature response via H3K27me3 and H2A.Z in Arabidopsis thaliana
}

Yogendra Bordiya ${ }^{1 *}$, Junghyun Kim ${ }^{1 *}$, Yanpeng $\mathrm{Xi}^{1}$, Dong-Hwan Kim ${ }^{1, \#}$, Youngjae Pyo ${ }^{1, \#}$, Bo Zhao $^{1}$, Wei Zong ${ }^{1}$, William A. Ricci ${ }^{2}$, Xiaoyu Zhang ${ }^{2}$, Sibum Sung ${ }^{1, \$}$

Affiliations:

${ }^{1}$ Department of Molecular Biosciences, Institute for Cellular \& Molecular Biology, The University of Texas at Austin, Austin, TX 78712, USA

${ }^{2}$ Department of Plant Biology, University of Georgia, Athens, GA 30602, USA

\# Current addresses:

D.-H. Kim: Department of Plant Science and Technology, Chung-Ang University, Anseong 17546, Republic of Korea

Y. Pyo: Department of Radiation Biology, Korea Atomic Energy Research Institute, Daejeon 34057, Republic of Korea

* These authors equally contribute to the manuscript.

${ }^{\$}$ Correspondence should be addressed to:

Sibum Sung, sbsung@austin.utexas.edu 


\begin{abstract}
Adapting to the everchanging environment is key to a successful life for an organism. Eukaryotes reprogram their transcriptome in order to adapt to an unfavorable environment. To achieve this reprogramming, plants and animals employ multiple responses including epigenetic regulation. In the search for mutations compromised in high ambient temperature response, we found that VIL1, a PHD finger protein displays aberrant development at high temperature. RNA-seq analysis shows that vil1 fails to downregulate heat suppressed genes. H2A.Z ChIP-seq showed that unlike wild type, vil1 fails to evict H2A.Z from heat responsive genes. We also found that vil1 suppresses constitutive thermo-morphogenic phenotype of arp6. Supporting this phenotype, RNA-seq analysis revealed that constitutive heat responsive transcriptome of arp6 reverted back to the wild-type levels in arp6vil1. This observation suggests an antagonistic relationship between VIL1 and ARP6. We found that this antagonism can be explained in part by interaction between H3K27me3 and H2A.Z.
\end{abstract}

\title{
Keywords:
}

- Climate change

- Thermo-morphogenesis

- VIL1 (VERNALIZATION INSENSITIVE 3 LIKE 1)

- H3K27me3

- $\quad$ PRC2 (Polycomb Repressive Complex 2)

- ARP6 (Actin related protein 6)

- H2A.Z

- SWR1 complex 


\section{Introduction}

Normal development is the first step towards completing a successful life cycle. The ability of organisms to grow, reproduce and achieve their maximum physical potential can be severely affected by the environmental perturbations. Changes in the climate are leading to widespread variation in important environmental factors such as temperature. The average global temperature on Earth has increased by about $0.6^{\circ} \mathrm{C}$ in the last century ${ }^{1}$. Most of this warming has occurred in the past three decades at the rate of $0.15-0.20^{\circ} \mathrm{C}$ per decade. Increase in global temperature caused by climate change will have a devastating effect on biodiversity, crop yield and ultimately on human health ${ }^{2-6}$. Adapting to the everchanging environment is key to the successful life of an organism and ultimately of a species. To cope with unfavorable conditions, eukaryotes employ multiple responses including epigenetic regulation. These epigenetic regulations help in reprogramming of the transcriptome to cope with varying conditions. This process requires conversion of the environmental stimuli to a molecular signal. Much progress has been made to identify receptors to these sorts of environmental stimuli. Studies have also implicated the role of histone variant $\mathrm{H} 2 \mathrm{~A} . \mathrm{Z}$ in mediating the thermo-sensory response in plants. An evolutionarily conserved SWR1 complex deposits histone variant H2A.Z and regulates multiple developmental processes in eukaryotes ${ }^{7}$. Multiple reports have shown that H2A.Z is evicted from the histone octamer to be replaced with canonical $\mathrm{H} 2 \mathrm{~A}$ in response to high ambient temperature, however the molecular mechanism behind this eviction remains unknown.

To identify additional regulators of ambient temperature responses, we took reverse genetic approach to uncover novel epigenetic components involved in response to high ambient temperature. Namely, we screened a number of mutants previously shown to be involved in the chromatin modifications. Surprisingly, we identified that mutations in VIL1 (VIN3-LIKE 1)/VERNALIZATION 5(VRN5) impair high ambient temperature responses. VIL1, a PRC2 (Polycomb Repression Complex 2) associated protein, is known to mediate a cold-temperature 
response, vernalization ${ }^{8,9}$. Previous studies also implicated the role of a component of the SWR1 complex in mediating temperature response ${ }^{10-16}$. This prompted us to investigate the mechanisms by which temperature-triggered responses are mediated through both PRC2 and SWR1. We found that H2A.Z's thermo-sensory response is influenced by the function of VIL1. Here, we show that VIL1 is required for response to warm ambient temperature because of two main factors: 1) by downregulating the heat suppressed genes through its PRC2 associated function, and; 2) by evicting $\mathrm{H} 2 \mathrm{~A} . \mathrm{Z}$ in from heat responsive genes. 


\section{Results}

\section{vil1 is hyposensitive to high ambient temperature}

We took reverse genetic approach to uncover novel player in high ambient temperature in plants. For this purpose, we screened an array of mutants of genes ranging from histone readers, writers, erasers, chromatin remodelers to histone variants and found that mutation in VIL1, a PRC2 associated protein, displayed aberrant development at high ambient temperature of $27^{\circ} \mathrm{C}$ compared to WT (Fig. 1, Supplementary Fig S1). VIL1 is a PHD finger domain containing protein which has been shown to participate in both the photoperiod and vernalization flowering pathways in Arabidopsis by regulating expression of the related floral repressors FLOWERING LOCUS C and FLOWERING LOCUS $M^{8}$. PRC2 biochemically copurifies with members of the VERNALIZATION INSENSITIVE3 (VIN3) family of proteins, including VIN3, VIL1 and VIL2 ${ }^{17,18 .}$ All VIN3 family proteins including VIL1 are known to act together with PRC2 to increase repressive histone marks at floral repressor loci in Arabidopsis ${ }^{18}$. In order to adapt to the high temperature environment, plants undergo thermo-morphogenesis. Thermo-morphogenesis is morphological and architectural changes induced by high ambient temperatures ${ }^{19-21}$. Thermo-morphogenesis at elevated ambient temperature involves changes in plant architecture such as elongation of hypocotyl in seedlings and extended petiole length and hyponasty in the rosette to keep plant body well above hot ground and facilitate aeration ${ }^{19}$. We found that unlike WT, vil1 does not show extended petiole length (Fig. 1A). Moreover, when switched from $22^{\circ} \mathrm{C}$ to $27^{\circ} \mathrm{C}$ growth condition, relative hypocotyl lengthening was reduced in vil1 (Supp. Fig. 1B and C). It is very well established that thermo-morphogenic phenotype of long hypocotyl and petiole length is achieved with the help of auxin ${ }^{22-25}$. Consistent with this, the inducibility of auxin responsive genes like YUC8 and IAA29 has been compromised in vil1 (Supp Fig. 1D). This poor response in terms of phenotype suggests that vil1 is hyposensitive to warm temperature. We also checked the flowering time data and 
found that vil1 displayed late flowering phenotype in short day condition compared to WT both at $22^{\circ} \mathrm{C}$ and $27^{\circ} \mathrm{C}$ (Fig. 2D).

\section{Transcriptional changes upon high ambient temperature are compromised in vil1 mutants}

To address the molecular basis of hyposensitivity of vil1 towards high temperature, we performed RNA-seq. First, we performed an overlap analysis between genes up and downregulated in WT and vil1 at $22^{\circ} \mathrm{C}$ vs $27^{\circ} \mathrm{C}$ and found that there are 5,061 genes up- and down- regulated in response to high temperature in WT, whereas only 910 genes are up and down regulated in vil1 (Fig. 1B). Heatmap analysis shows that vil1 fails to downregulate majority of heat suppressed genes and also fails to upregulate a number of heat induced genes (Fig. 1C). In order to find specific genes responsible for hyposensitivity of vil1 to high temperature, we performed hierarchical clustering analysis. Out of 17 clusters, clusters 5 and 17 contain genes which are not induced at high temperature as much as WT in vil1 (Fig. 1D). GO (Gene Ontology) term analysis of these clusters showed that clusters 5 and 17 contain genes responsive to heat and stress (Supp Fig. 1A). Cluster 7 , on the other hand contains genes which are suppressed by high temperature in WT but not in vil1. GO term analysis of cluster 7 shows enrichment of genes related to photosynthesis and response abiotic stimulus. It has been reported that photosynthetic activity is reduced at high ambient temperature in order to cope with the stress ${ }^{26}$. These results also suggest that vil1 fails to reduce photosynthetic activity at higher temperature unlike WT. RPKM normalized gene count from all RNA-seq samples is provided in supplementary table 3. Taken together, all these results suggest that vil1 is hyposensitive in high ambient temperature in terms of morphological responses as well as of transcriptomic responses.

\section{vil1 suppresses constitutive thermo-morphogenic phenotype of arp6}


Switching from canonical histone to a non-canonical variant from histone octamer is a well-studied mode of chromatin remodeling ${ }^{27}$. In Arabidopsis, a component of the SWR1 complex, ARP6 (Actin Related Protein 6), is involved in the deposition of histone variant H2A.Z ${ }^{28}$. Loss of ARP6 in Arabidopsis leads to a major developmental and pleiotropic phenotype ${ }^{29}$. H2A.Z has been implicated to play an important role in not only developmental regulation but also in the biotic and abiotic stress response ${ }^{10,30-32}$. Recently, multiple reports implicated the role of H2A.Z in mediating thermo-sensory response in plants ${ }^{11-13}$. Interestingly, previous studies have shown that the wildtype plants grown at higher ambient temperature $\left(27^{\circ} \mathrm{C}\right)$ and $\operatorname{arp} 6$ plants grown at normal ambient temperature $\left(22^{\circ} \mathrm{C}\right)$ show a similar phenotype and transcriptome profile ${ }^{11}$. This indicates that H2A.Z is a major regulator of the heat response in Arabidopsis. Remarkably, the PIF4 mediated thermo-morphogenesis requires removal of histone variant H2A.Z from the histone octamer at promoters of growth related genes ${ }^{14}$. This also explains why arp6 plants grown at $22^{\circ} \mathrm{C}$ phenocopy wild-type plants grown at $27^{\circ} \mathrm{C}$.

It is known that under high ambient temperature and stress, plants accelerate the development and transition to flowering to be able to reproduce in time ${ }^{33}$. arp6 shows constitutive thermomorphogenic phenotype and accelerated flowering (Fig. 2A) resulting from the constitutive expression of heat responsive genes ${ }^{11}$. Consistently, arp6 also shows longer petiole length at $22^{\circ} \mathrm{C}$ compared to WT (Fig. 2B and $2 \mathrm{C}$ ). Strikingly, we found that vil1 suppresses constitutive thermo-morphogenic phenotype of arp6 suggesting that VIL1 and ARP6 have antagonistic functions (Fig. 2A and 2B). Unlike arp6 single mutant, arp6/vil1 double mutant no longer show long petiole length and early flowering phenotype associated with high ambient temperature (Fig. 2C and 2D). We confirmed this double mutant phenotype using another allele of vil1 (vil1-2) (Supp. Fig. 5A). 
RNA-seq analysis supports suppression of thermo-morphogenic phenotype of arp6-1/vil11 double mutant and reveals antagonistic relationship between VIL1 and ARP6

In order to find out if the transcriptome supports antagonistic relationship between VIL1 and ARP6, we performed RNA-seq in Col-0 (WT), vil1, arp6, and arp6vil1. To confirm the previously published result that arp6 at normal ambient temperature of $22^{\circ} \mathrm{C}$ displays a transcriptome similar to that of a plant grown at higher ambient temperature of $27^{\circ} \mathrm{C}^{11}$, we performed a correlation analysis. For this purpose, we compared the transcriptome of WT plants grown at $27^{\circ} \mathrm{C}$ and arp6 plants grown at $22^{\circ} \mathrm{C}$. The result shows a clear positive correlation (Fig. $3 \mathrm{~A}$ ) confirming conclusion from previous studies. Note that all the transcribed genes in the genome were used for this correlation analysis. Since vil1 is hyposensitive to high ambient temperature, when we compared the transcriptome of vil1 plants grown at $27^{\circ} \mathrm{C}$ and arp 6 plants grown at $22^{\circ} \mathrm{C}$, as expected, the positive correlation is lost to a great extent (Fig. 3B). Next, we took the similar approach considering only the genes which are up- and down-regulated in response to heat in WT and performed a boxplot analysis. This analysis elegantly captures the hyposensitivity of vil 1 and hypersensitivity of arp6 to high temperature (Fig. 3C). Remarkably, exaggerated constitutive heat responsive transcriptome of arp6 reverted back to the wild-type levels in arp6vil1 (Fig. 3C). This result is consistent with the reversed morphological phenotype of arp6vil1 compared to arp6 which includes not only the change in flowering time but also unlike arp6 single mutant, arp6/vil1 double mutant no longer show long petiole length phenotype associated with high ambient temperature (Fig. 2A, 2B, 2C and 2D). We further confirmed this phenotype using another allele of vil1 and via genetic complementation (Supp Fig. 5B). Next, to check if the reversal in the transcriptome in arp6vil1 is limited to the heat responsive genes or to the overall transcriptome, we performed the correlation analysis of transcripts between WT and arp6vil1 and found highly significant correlation $\left(R^{2}=0.85\right)$. This result suggest that majority of the transcripts differentially expressed in arp6 compared to wild-type revert back to the wild-type level in arp6vil1 (Fig. 3E). The top panel 
of supplementary figure 2 shows IGV screenshots of RNA-seq data showing reversion of transcriptome in arp6vil1. We confirmed the RNA-seq data with qRT-PCR on randomly selected genes on independent samples (Supp. Fig. 2). These results led us to the hypothesis that VIL1 and histone variant H2A.Z have antagonistic function in the regulation of gene expression in Arabidopsis.

\section{Clustering analysis reveal genes reversed in arp6/vil1}

To find out how many and what kind of genes are reversed in arp6vil1, we performed hierarchical clustering of RNA-seq in Col-0 (wild-type), vil1, arp6, and arp6vil1 followed by GO term analysis on these clusters. Out of ten clusters, clusters 1 and 2 with 3,797 and 3,840 genes respectively contain genes which are up and downregulated in arp6 compared to the WT, however, their expression was back to the WT level in arp6vil1 double mutant (Fig. 4A). The heatmap of these clusters is shown in Fig. 4B. In order to find out biological relevance behind this antagonism between VIL1 and ARP6 we performed GO term analysis on these clusters. No clear term to explain the relationship was found.

\section{Overall H2A.Z level remains unchanged in vil1-1 compared to WT}

We have two hypotheses to explain the antagonistic relationship between ARP6 and VIL1; 1) reduced deposition of H2A.Z in arp6 and enhanced deposition of H2A.Z in vil1 or: 2) Interaction between histone modifications, such as $\mathrm{H} 3 \mathrm{~K} 27 \mathrm{me} 3$, and $\mathrm{H} 2 \mathrm{~A} . \mathrm{Z}$ have antagonistic roles in regulation of gene expression. To check the first possibility, we performed the H2A.Z ChIP-seq at $22^{\circ} \mathrm{C}$ and in WT, vil1, arp6 and arp6vil1. MACS2 ChIP-peak analysis revealed about 16,000 peaks in both WT and vil1 (Supp Table 2) suggesting that there was no peak loss or gain in vil1. We then checked the relative enrichment of the H2A.Z using deepTools among four genotypes. As expected, the enrichment of H2A.Z was poor in arp6 compared to the WT, however, there was no significant change between WT and vil1 and between arp6 and arp6vil1 (Fig. 5A). To find out 
the basis of antagonism between ARP6 and VIL1, we did the H2A.Z enrichment analysis in clusters 1 and 2 which contain genes reversed in arp6vil1. We did not find significant difference between WT and vil1 and between arp6 and arp6vil1 in both clusters (Fig. 5B). These results suggest that overall H2A.Z level remains unchanged in vil1 compared to WT and that the antagonisms between VIL1 and ARP6 cannot be explained by H2A.Z enrichment alone.

\section{vil1-1 fails to evict $\mathrm{H} 2 \mathrm{~A} . \mathrm{Z}$ from high ambient temperature responsive genes}

Given the role of $\mathrm{H} 2 \mathrm{~A} . \mathrm{Z}$ in thermo-sensory response in Arabidopsis and genetic interaction between VIL1 and ARP6, we decided to perform H2A.Z ChIP-seq at $22^{\circ} \mathrm{C}$ and $27^{\circ} \mathrm{C}$ WT and vil1. As indicated in the previous section, at the global level, there was no significant change in the enrichment of $\mathrm{H} 2 \mathrm{~A} . \mathrm{Z}$ between WT and vil1 at $22^{\circ} \mathrm{C}$. To look at the $\mathrm{H} 2 \mathrm{~A} . \mathrm{Z}$ level in heat responsive genes specifically, we performed the differential binding analysis using Diffbind tool to compare the $\mathrm{H} 2 \mathrm{~A} . \mathrm{Z}$ fold change between $22^{\circ} \mathrm{C}$ and $27^{\circ} \mathrm{C}$. As reported in a number of studies, $\mathrm{H} 2 \mathrm{~A} . \mathrm{Z}$ level reduced at $27^{\circ} \mathrm{C}$ compared to $22^{\circ} \mathrm{C}$ in WT (Fig. 6A), however, the level remained high in case of vil1 at $27^{\circ} \mathrm{C}$ (Fig. 6B). To confirm that this change in level of $\mathrm{H} 2 \mathrm{~A} . \mathrm{Z}$ at $27^{\circ} \mathrm{C}$ is correlated with change in gene expression, we looked at the RNA expression level change (Fig. 6C). We found that indeed the level of H2A.Z in heat responsive genes was reduced in WT and that this reduction was not seen in vil1 leading to poor induction of heat responsive genes (Fig. 6C). When we looked at more heat responsive genes, similar pattern was found (Supp. Fig. 3). This result suggests that VIL1 is required for the eviction of $\mathrm{H} 2 \mathrm{~A} . \mathrm{Z}$ from heat responsive genes. It also explains in part the hyposensitive nature of vil1 mutants at high ambient temperature.

\section{Antagonism between VIL1 and ARP6 in part can be explained by H3K27me3}

We performed ChIP-qPCR on various histone modifications to check the possibility of interaction between H2A.Z and histone modifications. Studies suggest that the interaction between H2A.Z 
and PRC2 might be much more complex and involve additional histone modifications. Here we tried to examine relationship of $\mathrm{H} 3 \mathrm{~K} 27 \mathrm{me} 3, \mathrm{H} 3 \mathrm{~K} 4 \mathrm{me} 2$ and $\mathrm{H} 3 \mathrm{~K} 4 \mathrm{me} 3$ and other modifications with H2A.Z. For this purpose, we randomly selected transcriptionally reversed genes in arp6vil1 and performed ChIP-qPCR in WT, vil1, arp6 and arp6vil1 backgrounds. IGV snapshot of these genes is shown in Fig. 7A. We performed ChIP-qPCR to check H3K27me3, H3K9me2, H3K4me2, H3K4me3, H3 acetylation and RNA Pol II (S5). Interestingly, we did not find significant change in any of these histone modifications and RNA Pol II enrichment except for H3K27me3 (Fig. 7B and Supp Fig. 4). It's clear that H3K27me3 level in low in vil1 compared to WT which is expected given VIL1 is a PRC2 associated protein. Interestingly, the level of H3K27me3 was further reduced in arp6vil1 double mutant. To gain more insight into the role of H3K27me3 in antagonism between VIL1 and ARP6, we performed overlap analysis between transcriptionally reversed genes in arp6vil1 (clusters 1 and 2) and H3K27me3 enriched genes in Arabidopsis genome. 27.2\% $(1,045)$ genes from cluster 2 were enriched with H3K27me3 as opposed to mere $5.4 \%$ from cluster 1 (Fig. 7C). When we looked at the proportion of 1045 genes up and downregulated in vil1, arp6, and arp6vil1 compared to WT, while all of these genes were downregulated in arp6, about $75 \%$ reversed back to upregulation in arp6vil1 (Fig. 7D). To check if VIL1 binds at these 1045 reversed loci, we randomly selected 7 genes for VIL1 ChIP-qPCR. Except for AT4G29100 which was used as negative control, all other loci show VIL1 enrichment including FLC (Supp. Fig. 7).

Our results clearly show that VIL1 plays an important role in warm temperature response in Arabidopsis through its function in imparting H3K27me3 and removal of H2A.Z. To support this, we also show a strong genetic interaction/antagonism between VIL1 and ARP6. This study provides a genetic basis of the interaction between chromatin modifications and histone variants to mediate responses to environmental changes.

\section{Discussion}


In this study we reveal a new player in the high ambient temperature response. Role of PRC2 in vernalization in response to cold and in other plant developments has been widely studied and well established. Our findings provide new insight on the role of PRC2 associated protein during development at high temperatures. PRC2 plays an important role not only in the growth, development and differentiation ${ }^{34}$ but also in response to stress ${ }^{35}$. It is interesting to note that VIL1 was originally isolated as vernalization insensitive mutants which mediate cold temperature response. The same protein also plays role in the other spectrum of temperatures, high ambient temperature. Our result also implies that interaction between H2A.Z and H3K27me3/PRC2 dramatically affect the gene expression.

During this study, we found an antagonistic relationship between VIL1-mediated H3K27me3 and SWR1 complex-mediated H2AZ deposition. Broadly speaking, this reflects antagonism between chromatin modifications. A myriad of histone modifications coexist on the chromatin, studying how presence of one affect another is very important to understand how transcription is regulated in Eukaryotes. Extensive research in embryonic stem cells (ESCs) suggests that H2A.Z preferentially localizes to promoters of inactive genes occupied by the PRC2 ${ }^{36,37}$. In addition, H2A.Z is also enriched at enhancers in ESCs and facilitates the binding of PRC2 and H3K4me3 and H3K27me3 modifications ${ }^{38}$. Such enhancers have also been referred to as poised enhancers. These studies suggest that the interaction between H2A.Z and PRC2 might be much more complex and involve additional histone modifications. A previous study in Arabidopsis utilized pure bioinformatics approach to study the relationship between histone variant $H 2 A . Z$ and histone modifications such as $\mathrm{H} 3 \mathrm{~K} 27 \mathrm{me} 3$ and $\mathrm{H} 3 \mathrm{~K} 4 \mathrm{me} 3{ }^{39}$. However, to this date there is no report showing genetic evidence of interaction between components of PRC2 and that of SWR1 complex in plants. To confirm that this antagonism is not an indirect effect of the large pleiotropic changes in arp6 genetic background, we also generated vil1/hta9/hta11 triple mutant. HTA8, HTA9 and HTA11 are three genes in Arabidopsis which code of histone variant H2A.Z. hta9/hta11 
double mutant phenocopy arp6. As expected, vil1 suppresses the early flowering phenotype of hta9/hta11 and the overall phenotype of vil1/hta9/hta11 triple mutant resembles more like arp6vil1 (Supp Fig. 6). Wang et al. showed interaction among H2A.Z, H3.3 and PRC2-dependent $\mathrm{H} 3 \mathrm{~K} 27 \mathrm{me} 3$ in mouse embryonic stem cells ${ }^{40}$. They suggest that H3.3 counteracts the action of H2A.Z. It is possible that VIL1 or PRC2 indirectly affect the level of H3.3 in the Arabidopsis genome leading the antagonism between these modifications.

We ascribe the hyposensitivity of vil1 at high ambient temperature to two main factors; 1) lack of H3K27me3, because vil1 fails to downregulate the heat suppressed genes, and: 2) lack of H2A.Z eviction in vil1 in from heat responsive genes. Given these two reasons, it is not surprising that vil1 and arp6 show a strong genetic interaction. It is likely that VIL1 or PRC2 by extension could be involved in eviction of H2A.Z in Arabidopsis. Although it is beyond the scope of this study, to address this possibility, the interaction between PRC2 associated proteins and INO80 complex needs to be addressed. INO80 is required for maintaining the boundary of $\mathrm{H} 2 \mathrm{~A} . \mathrm{Z}$ at +1 nucleosome by evicting the ectopically deposited H2A.Z by SWR1 complex ${ }^{41}$.

Our study enhances our understanding of how eukaryotes utilize epigenetic mechanisms under unfavorable environmental conditions. The insights developed from such research will lead us to develop better strategies to thrive in the era of climate change. 


\section{Acknowledgements}

The authors acknowledge the Texas Advanced Computing Center (TACC; http://www.tacc.utexas.edu) at The University of Texas at Austin for providing High Performance Computing resources that have contributed to the research results reported within this paper. This work was supported by NIH R01GM100108 and NSF IOS 1656764 to S. S. 


\section{Materials and methods}

\section{Plant material and growth conditions}

All experiments were done in Col-0 ecotype of Arabidopsis thaliana. The Arabidopsis T-DNA lines used in this research are: vil1-1 (SALK_136506), vil1-2 (SALK_140132), arp6-1 (Garlic_599_G03), hta9-1 (SALK_054814), hta11-1 (SALK_017235). Plants were grown in long day (16-hour light, 8-hour dark) and short day (8-hour light, 16 -hour dark) at $22^{\circ} \mathrm{C}$ or $27^{\circ} \mathrm{C}$. VIL1::gVIL1-myc complementation line in vil1-1 mutant background was used for VIL1 ChIPqPCR. Experiment specific growth condition is also mentioned in the figure legend and/or results section. For heat treatment for RNA-seq, seedlings were germinated and grown on blue media (fertilizer) for 7 days at $22 \mathrm{C}$ followed by 9 days at $27^{\circ} \mathrm{C} .22^{\circ} \mathrm{C}$ samples were continuously grown at $22^{\circ} \mathrm{C}$ for 14 days. For all the experiments samples were harvested at ZT-6.

\section{RNA-seq}

Total RNA was extracted from seedlings (growth condition described above) by using TRIzol (Invitrogen) and treated with DNase I enzyme (Promega) to eliminate traces of genomic DNA. Sequencing libraries were prepared with $500 \mathrm{ng}$ total RNA followed by NEBNext Poly(A) mRNA Magnetic Isolation and library preparation using NEB \#E7420. Quality of library was assessed using Bioanalyzer (Agilent High Sensitivity DNA Assay). All the sequencing was performed on Illumina NextSeq 500 as 35X2 paired end.

For bioinformatics analysis reads generated by Illumina NextSeq 500 platform were checked for quality using FastQC. After quality assessment, reads were aligned on TAIR10 genome using HISAT2. SAM files generated from mapping were then converted into BAM files and sorted using Samtools. Bigwig files for IGV visualization were created using deepTools. For the gene counting bedtools program was used which generates raw count using BAM files. Raw count was then fed 
into $\mathrm{R}$ for differential gene expression analysis using edgeR and data visualization. For hierarchical clustering analysis only differentially expressed genes were used. Specific analysis detail is also provided in figure legend. Codes for the analysis can be provided upon request. Go term enrichment analysis was performed using Gene Ontology website http://geneontology.org/.

\section{H2A.Z ChIP-seq}

Seedlings were crosslinked using $1 \%$ formaldehyde. ChIP procedure was followed as described in ${ }^{42}$. Library was prepared from immunoprecipitated and input samples using NEBNext ChIP-Seq Library Prep Master Mix Set for illumine (E6200L). Quality of library was assessed using Bioanalyzer (Agilent High Sensitivity DNA Assay). For bioinformatics analysis reads generated by Illumina NextSeq 500 platform were checked for quality using FastQC. After quality assessment, reads were aligned on TAIR10 genome using Bowtie2. SAM files generated from mapping were then converted into BAM files and sorted using Samtools. Bigwig files for IGV visualization were created using deeptools. MACS2 broad peak call was used for peak calling $(q<0.01)$. For differential binding analysis, DiffBind package was used in R and MA plots were generated using the same package. For H2A.Z enrichment analysis, deepTools package was used. First, computeMatrix was generated and then plotProfile function was used to generate the enrichment profiles.

\section{qRT-PCR}

RNA was extracted using TRIzol reagent (Ambion). $1 \mu \mathrm{g}$ total RNA was used to synthesize cDNA. After DNasel treatment to remove any DNA contamination, random primer mix (NEB \#S1330S) and M-MLV Reverse transcriptase (Invitrogen \#28025-013) were used for first strand synthesis. qRT-PCR was used to quantify the RNA prepared from transient expression experiments. AzuraQuant qPCR Master Mix (Azura Genomics) was used with initial incubation at $95^{\circ} \mathrm{C}$ for 2 min followed by 40 cycles of $95^{\circ} \mathrm{C}$ for $10 \mathrm{sec}$ and $60^{\circ} \mathrm{C}$ for $30 \mathrm{sec}$. Level of target RNA was 
calculated from the difference of threshold cycle $(\mathrm{Ct})$ values between reference and target gene. PP2A gene was used as reference. List of primers used for qRT-PCR is provided in supplementary table 1.

\section{ChIP-qPCR}

Seedlings were crosslinked using $1 \%$ formaldehyde. ChIP procedure was followed as described in ${ }^{42}$. Antibodies used for ChIP were ab1791 (H3), ab5408 (Pol II S5), ab1220 (H3K9me2), ab47915 (H3 acetylation), ab8580 (H3K4me3), ab11946 (H3K4me2) and Millipore 07-449 (H3K27me3). For gVIL1-myc ChIP anti c-Myc antibody was used (Santa Cruz Biotechnology \# 9E10). After ChIP, DNA was purified using Qiagen PCR purification kit (QIAquick 28106). AzuraQuant qPCR Master Mix (Azura Genomics) was used with initial incubation at $95^{\circ} \mathrm{C}$ for 2 min followed by 40 cycles of $95^{\circ} \mathrm{C}$ for $10 \mathrm{sec}$ and $60^{\circ} \mathrm{C}$ for 30 sec. For all histone ChIP-qPCR, H3 ChIP was used for normalization. List of primers used for ChIP-qPCR is provided in supplementary table 1. 


\title{
Supplementary information
}

\author{
Supplementary Figures S1 S7
}

Supplementary Tables S1 S3

Primers used for qRT-PCR and ChIP-qPCR (supplementary table 1)

H2A.Z ChIP-seq peak information (MACS2) (Supplementary table 2)

Raw read count and RPKM normalized RNA-seq count (Supplementary table 3) 
A

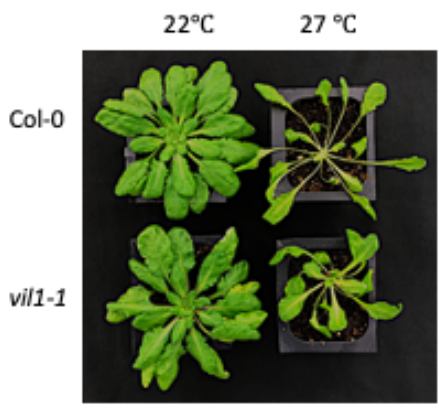

SD $\sim 7$ weeks old
B

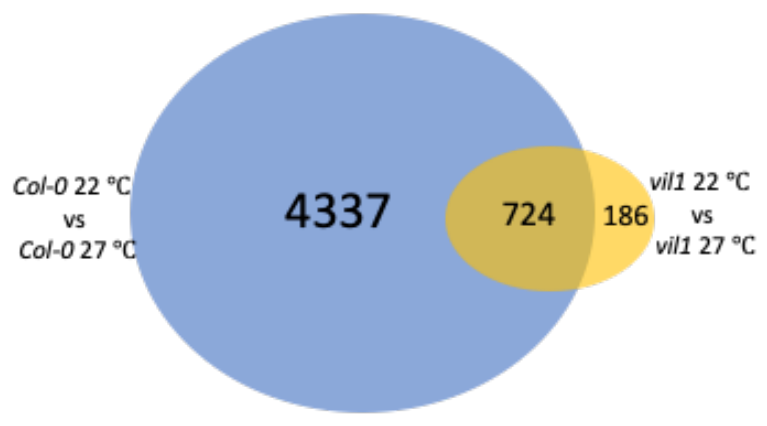

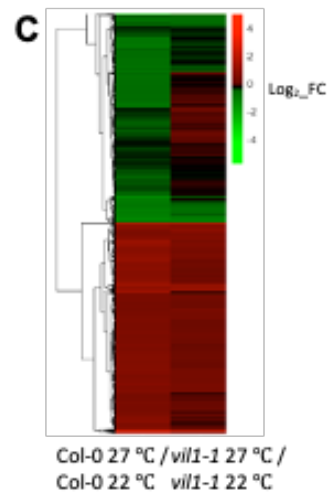

D

$\nabla N$ N $P$ T

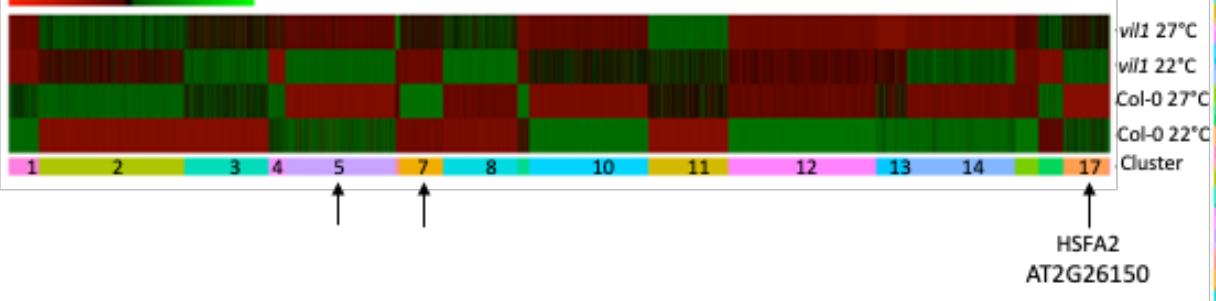

Figure 1. vil1 responds poorly to high ambient temperature and fails to undergo thermomorphogenesis

A. Col-0 (WT) and vil1-1 plants grown in short day condition continuously at either $22^{\circ} \mathrm{C}$ or $27^{\circ} \mathrm{C}$. As seen in the image, Col-0 undergo thermo-morphogenesis but vil1-1 does not. B. Venn diagram overlap analysis of up and downregulated genes in response to high temperature between Col-0 and vil1-1. Number of genes respond to high temperature in vil1-1 is much lower than in WT. C. Heatmap of up and downregulated genes show that vil1-1 fails to downregulate majority heat suppressed genes and fails to upregulate a number of genes at high temperature compared to WT. D. Differentially expressed genes were used for hierarchical clustering analysis in order to find which genes are affected in vil1-1. Clusters 5, 7 and 17 indicated by an arrow contain genes which respond poorly to temperature change in vil1-1. Cluster 17 contains heat shock transcription factor 2. GO term analysis of these clusters is in supplementary figure 1. 

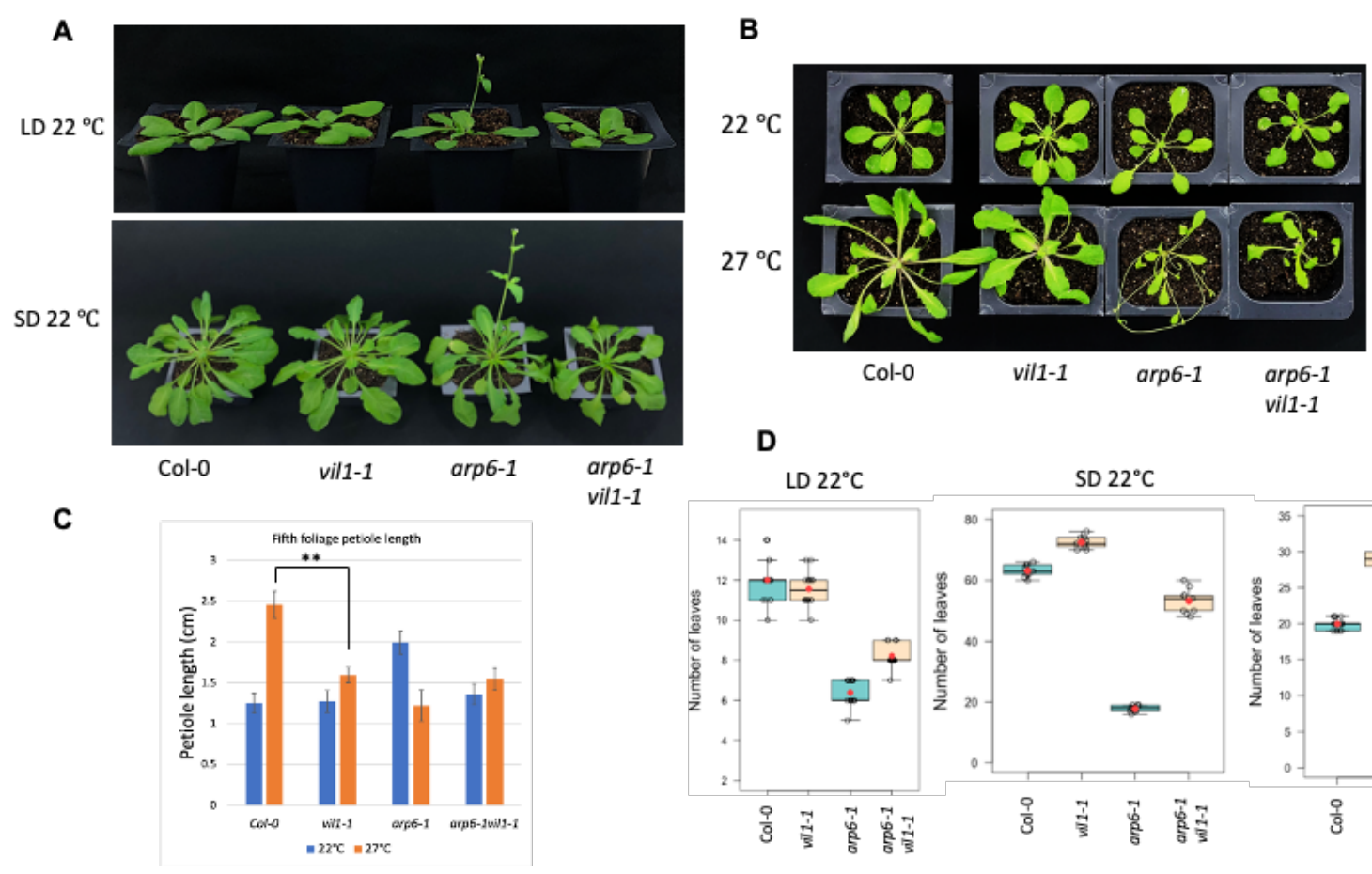

D

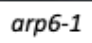
vil1-1

LD $22^{\circ} \mathrm{C}$

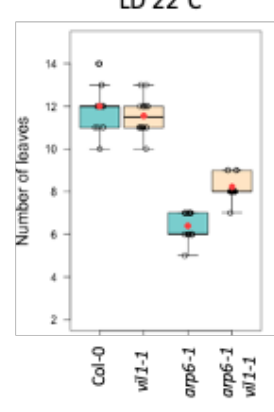

SD $22^{\circ} \mathrm{C}$

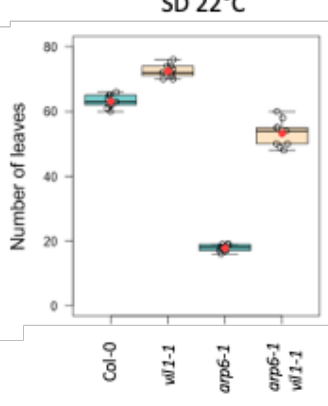

SD $27^{\circ} \mathrm{C}$

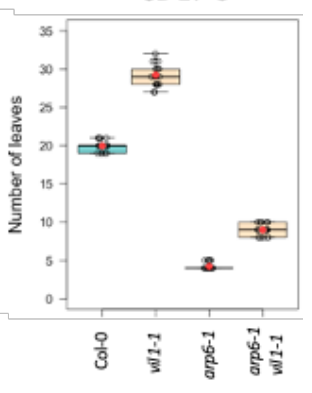

Figure 2. vil1-1 suppresses constitutive thermo-morphogenic phenotype of arp6-1

A. Both in long day (LD) and in short day (SD) conditions, early flowering and long petiole length phenotype of arp6 is suppressed by vil1 in arp6vil1. B and C. phenotype at $22^{\circ} \mathrm{C}$ and $27^{\circ} \mathrm{C}$ and the petiole length quantification data. Petiole length of fifth leaf is shown. D. Flowering time data shown in terms of number of leaves at the time of flowering. Red dot in the box plot indicate mean number of leaves. 

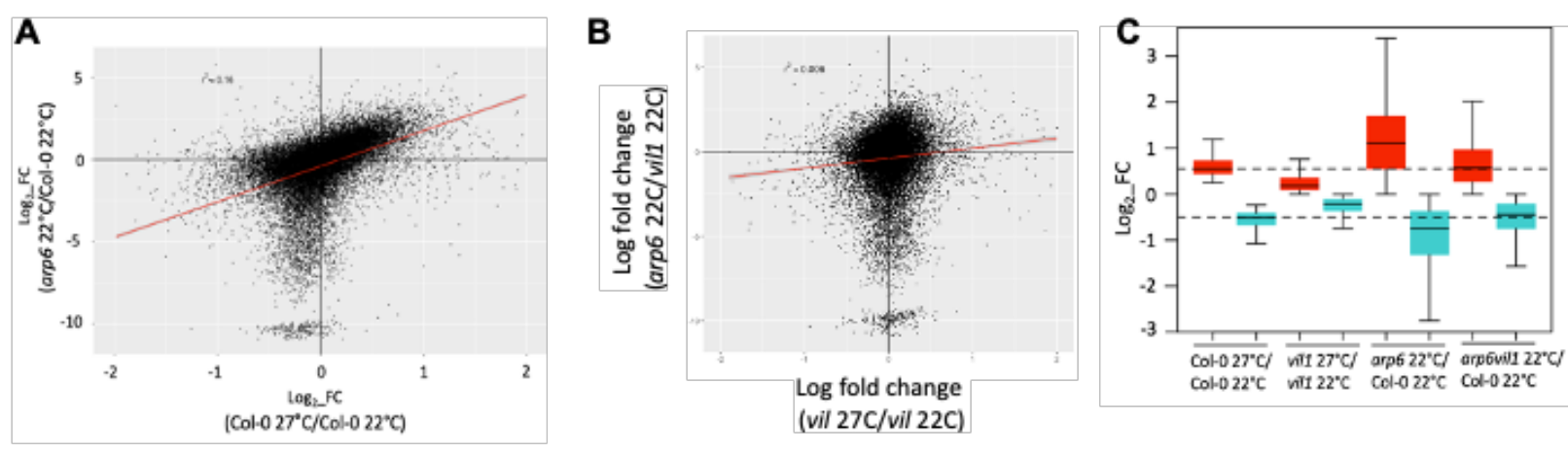

D
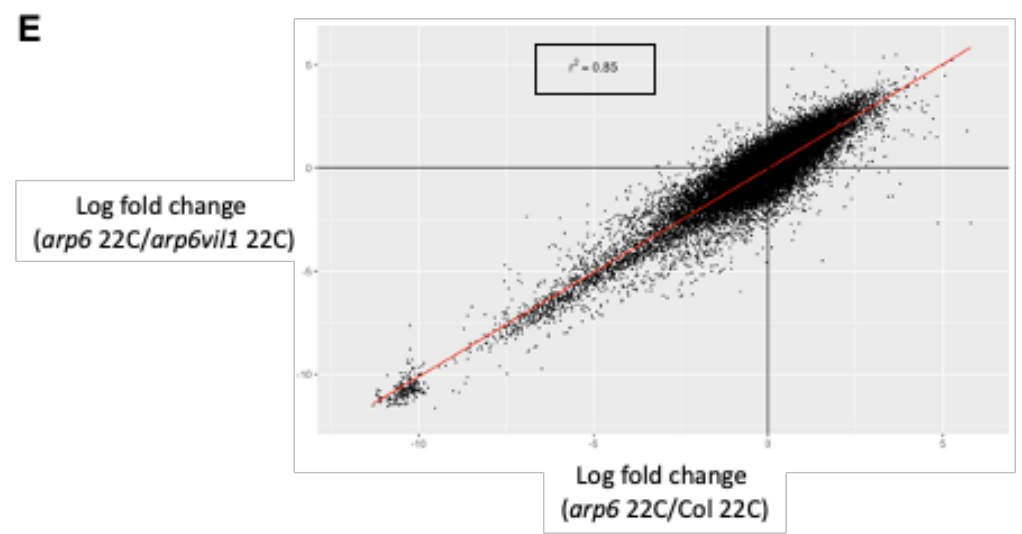

Figure 3. RNA-seq analysis supports suppression of thermo-morphogenic phenotype of arp6-1/vil1-1 double mutant and reveals antagonistic relationship between VIL1 and ARP6

A. Correlation analysis using all actively transcribed genes in Arabidopsis. Comparison of arp6 induced genome-wide transcriptional changes (arp6 $22^{\circ} \mathrm{C} / \mathrm{Col}-022^{\circ} \mathrm{C}$ ) with responses to increasing temperature from $22^{\circ} \mathrm{C}$ to $27^{\circ} \mathrm{C}$ in wild-type shows that transcripts upregulated in arp6 mutant at $22^{\circ} \mathrm{C}$ positively correlated with transcripts induced by temperature. B. The correlation is lost in the same comparison where WT is replaced with vil1. C. Box plot was created using differentially expressed genes at $27^{\circ} \mathrm{C}$ compared to $22^{\circ} \mathrm{C}$ in WT. Red color indicates upregulated genes and cyan color shows downregulated genes. Notice that when same genes were taken from arp6 $22 \mathrm{C}$ vs Col- $022^{\circ} \mathrm{C}$ comparison, they shown constitutive differential expression on heat responsive genes. D. Genes differentially expressed in arp6 compared to WT (arp6 $22^{\circ} \mathrm{C} / \mathrm{Col}-$ $022^{\circ} \mathrm{C}$ ) were used to create boxplot. These genes return to zero-fold change level in arp6vil1 $22^{\circ} \mathrm{C} / \mathrm{Col}-022^{\circ} \mathrm{C}$ comparison. E. All actively transcribed genes in Arabidopsis genome were used 
bioRxiv preprint doi: https://doi.org/10.1101/2020.04.29.069484; this version posted May 1, 2020. The copyright holder for this preprint (which was not certified by peer review) is the author/funder. All rights reserved. No reuse allowed without permission.

to create a scatter plot between the comparisons shown. Red line in all scatter plots represents linear regression line with $r^{2}$ value of 0.85 . FC (Fold Change). 


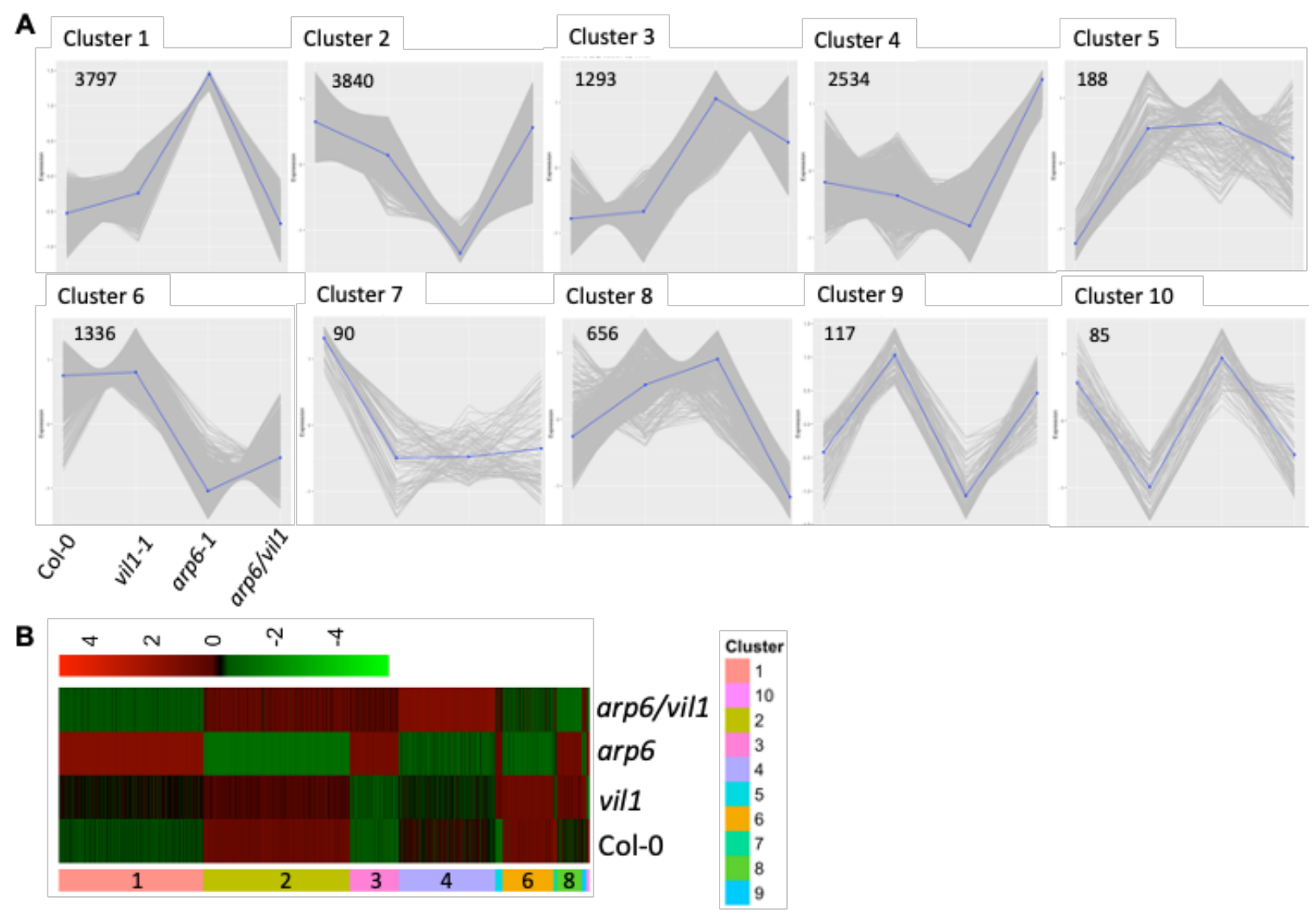

Figure 4. Clustering analysis reveal genes reversed in arp6/vil1

A. Differentially expressed genes were used for hierarchical clustering analysis in order to find which genes are transcriptionally reversed in arp6vil1. Out of 10 clusters, clusters 1 and 2 show transcriptionally reversed genes. B. Heatmap of 10 clusters. 
A

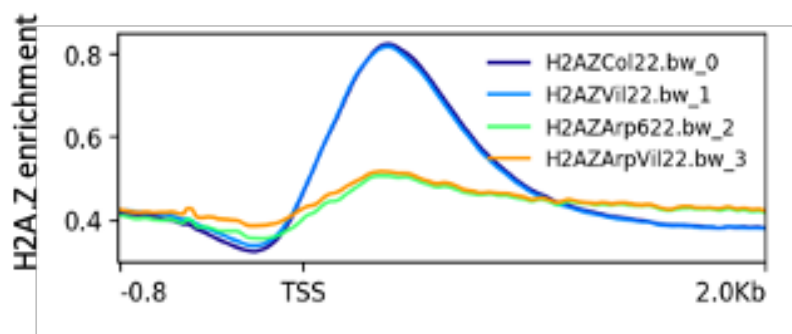

B
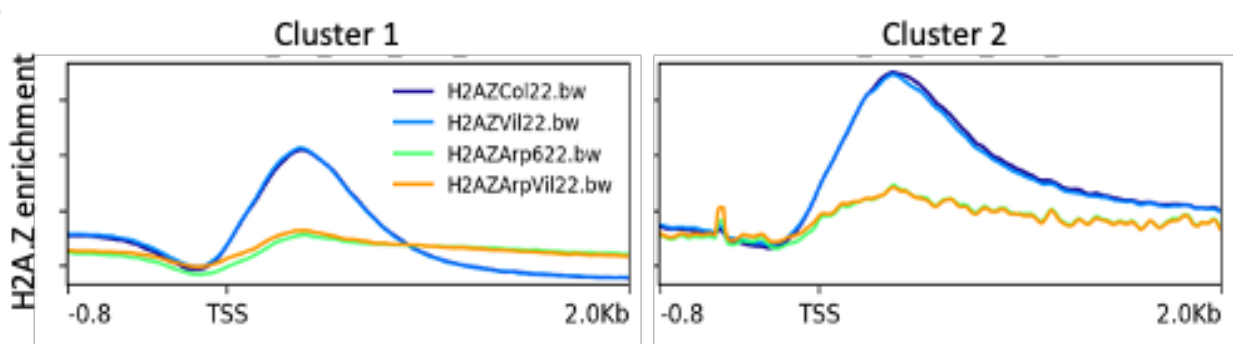

Figure 5. Overall H2A.Z level remain unchanged in vil1-1 compared to WT

BED file was created using MACS2 generated H2A.Z ChIP peaks information. This BED file was fed into deepTools to generate compute matrix followed by profile plotting to find the enrichment of H2A.Z from $800 \mathrm{bp}$ upstream of TSS to $2.0 \mathrm{kbp}$ downstream of genes. A. Profile of all the genome peaks comparing H2A.Z enrichment among WT, vil1, arp6 and arp6vil1. B. H2A.Z enrichment in genes from clusters 1 and 2 which are transcriptionally reversed in arp6vil1. 
A

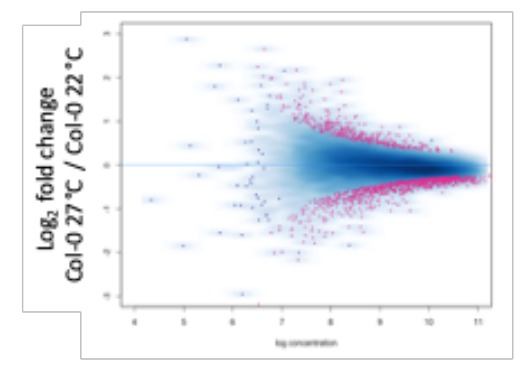

C

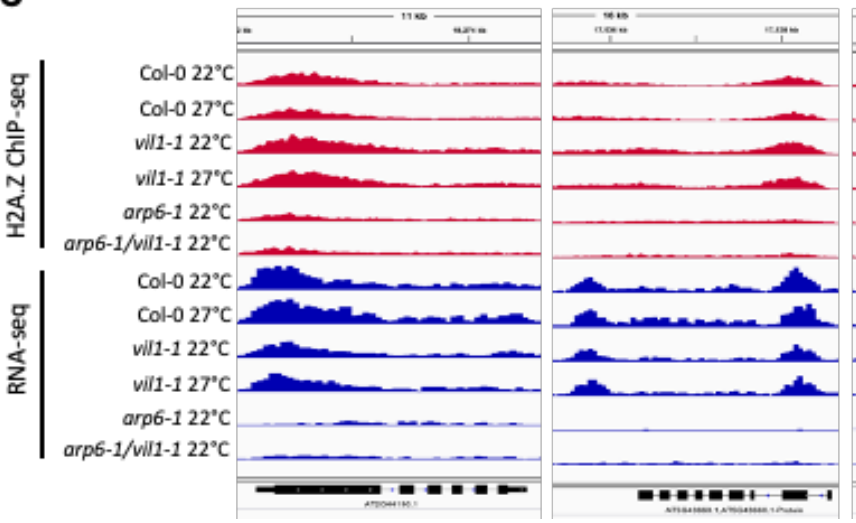

B
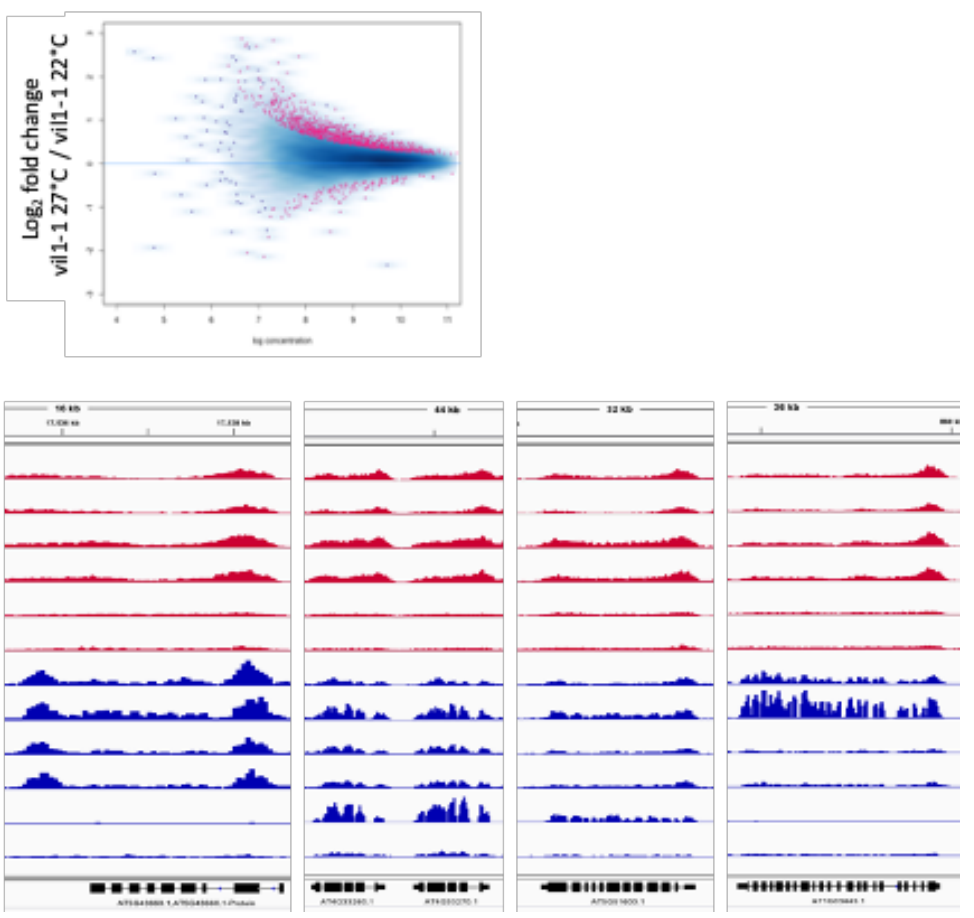

Figure 6. vil1-1 fails to evict H2A.Z from high ambient temperature responsive genes

A. MA plot with $\log _{2}$ fold change in the H2A.Z enrichment. Differential enrichment in Col-0 $27^{\circ} \mathrm{C} / \mathrm{Col}-022^{\circ} \mathrm{C}$ comparison and B. in vil1 $27^{\circ} \mathrm{C} /$ vil1 $22^{\circ} \mathrm{C}$ comparison. C. IGV snapshots with H2A.Z ChIP-seq and RNA-seq data of randomly selected genes which fail to evict H2A.Z in vil1 in response to high temperature. 
A

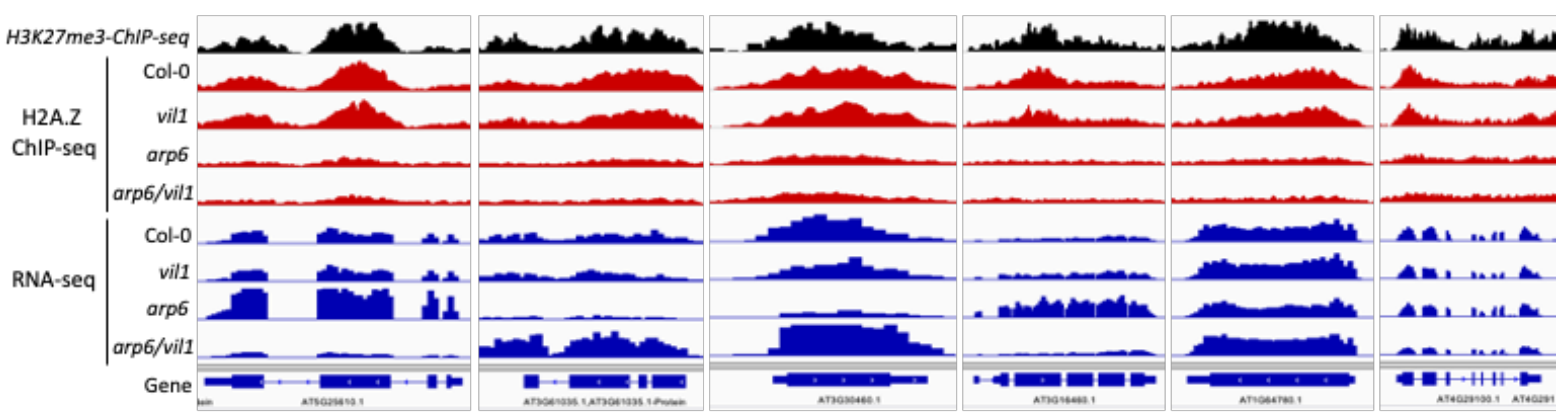

B

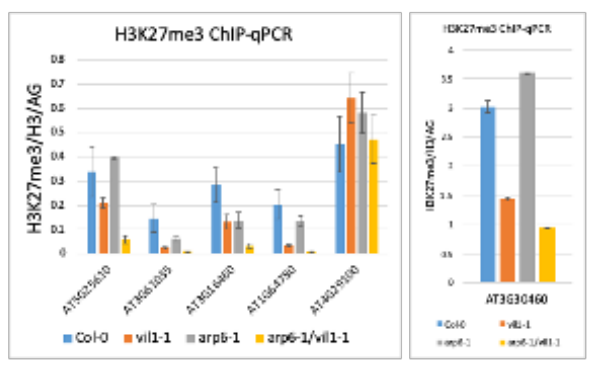

C

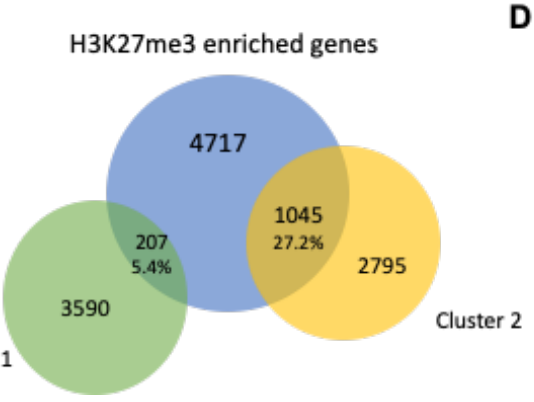

D

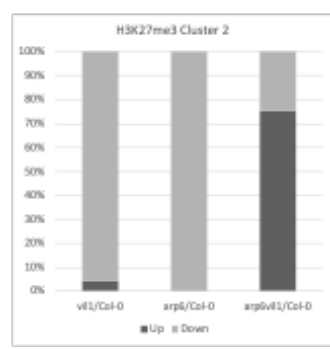

Figure 7. Antagonism between VIL1 and ARP6 in part can be explained by H3K27me3

A. Randomly selected transcriptionally reversed genes for ChIP-qPCR. B. H3K27me3 level normalized to $\mathrm{H} 3$ and Agamous gene. C. Overlap analysis of H3K27me3 enriched genes and cluster 1 and cluster 2 genes. D. Proportion of genes up and downregulated among 1045 genes which are common between H3K27me3 and cluster 2. 


\section{References}

1 Burnett, A. W., Kirby, M. E., Mullins, H. T. \& Patterson, W. P. Increasing Great Lake-effect snowfall during the twentieth century: A regional response to global warming? Journal of Climate 16, 3535-3542, doi:Doi 10.1175/1520-0442(2003)016<3535:Iglsdt>2.0.Co;2 (2003).

2 lizumi, T. et al. Responses of crop yield growth to global temperature and socioeconomic changes. Sci Rep 7, 7800, doi:10.1038/s41598-017-08214-4 (2017).

3 Holbrook, N. J. et al. A global assessment of marine heatwaves and their drivers. Nat Commun 10, 2624, doi:10.1038/s41467-019-10206-z (2019).

4 Smale, D. A. et al. Marine heatwaves threaten global biodiversity and the provision of ecosystem services. Nature Climate Change 9, 306-+, doi:10.1038/s41558-019-0412-1 (2019).

5 Chen, Y., Zhang, Z. \& Tao, F. L. Impacts of climate change and climate extremes on major crops productivity in China at a global warming of 1.5 and 2.0 degrees C. Earth System Dynamics 9, 543-562, doi:10.5194/esd-9-543-2018 (2018).

6 Tigchelaar, M., Battisti, D. S., Naylor, R. L. \& Ray, D. K. Future warming increases probability of globally synchronized maize production shocks. Proc Natl Acad Sci U S A 115, 6644-6649, doi:10.1073/pnas.1718031115 (2018).

7 Kobor, M. S. et al. A protein complex containing the conserved Swi2/Snf2-related ATPase Swr1p deposits histone variant H2A.Z into euchromatin. PLoS Biol 2, E131, doi:10.1371/journal.pbio.0020131 (2004).

8 Sung, S., Schmitz, R. J. \& Amasino, R. M. A PHD finger protein involved in both the vernalization and photoperiod pathways in Arabidopsis. Genes Dev 20, 3244-3248, doi:10.1101/gad.1493306 (2006).

9 Greb, T. et al. The PHD finger protein VRN5 functions in the epigenetic silencing of Arabidopsis FLC. Curr Biol 17, 73-78, doi:S0960-9822(06)02570-X [pii]

10.1016/j.cub.2006.11.052 (2007).

10 Kumar, S. V. H2A.Z at the Core of Transcriptional Regulation in Plants. Molecular Plant 11, 1112-1114, doi:https://doi.org/10.1016/j.molp.2018.07.002 (2018).

11 Kumar, S. V. \& Wigge, P. A. H2A.Z-Containing Nucleosomes Mediate the Thermosensory Response in Arabidopsis. Cell 140, 136-147, doi:https://doi.org/10.1016/j.cell.2009.11.006 (2010).

12 Wigge, P. A. Ambient temperature signalling in plants. Current Opinion in Plant Biology 16, 661-666, doi:https://doi.org/10.1016/j.pbi.2013.08.004 (2013).

13 Cortijo, S. et al. Transcriptional Regulation of the Ambient Temperature Response by H2A.Z Nucleosomes and HSF1 Transcription Factors in Arabidopsis. Molecular Plant 10, 1258-1273, doi:https://doi.org/10.1016/j.molp.2017.08.014 (2017).

14 Proveniers, M. C. G. \& van Zanten, M. High temperature acclimation through PIF4 signaling. Trends in Plant Science 18, 59-64, doi:https://doi.org/10.1016/j.tplants.2012.09.002 (2013).

15 Jean Finnegan, E. et al. Polycomb proteins regulate the quantitative induction of VERNALIZATION INSENSITIVE 3 in response to low temperatures. The Plant Journal 65, 382-391, doi:10.1111/j.1365-313X.2010.04428.x (2011). 
16 Sakamoto, T. \& Kimura, S. Plant Temperature Sensors. Sensors 18, 4365 (2018).

17 De Lucia, F., Crevillen, P., Jones, A. M., Greb, T. \& Dean, C. A PHD-polycomb repressive complex 2 triggers the epigenetic silencing of FLC during vernalization. Proc Natl Acad Sci U S A 105, 16831-16836, doi:10.1073/pnas.0808687105 (2008).

18 Kim, D.-H. \& Sung, S. Coordination of the Vernalization Response through a VIN3 and FLC Gene Family Regulatory Network in Arabidopsis. The Plant Cell 25, 454, doi:10.1105/tpc.112.104760 (2013).

19 Quint, M. et al. Molecular and genetic control of plant thermomorphogenesis. Nature Plants 2, 15190, doi:10.1038/nplants.2015.190 (2016).

20 Gangappa, S. N., Berriri, S. \& Kumar, S. V. PIF4 Coordinates Thermosensory Growth and Immunity in Arabidopsis. Curr Biol 27, 243-249, doi:10.1016/j.cub.2016.11.012 (2017).

21 Johansson, H. et al. Arabidopsis cell expansion is controlled by a photothermal switch. Nature Communications 5, 4848, doi:10.1038/ncomms5848

https://www.nature.com/articles/ncomms5848\#supplementary-information (2014).

22 Gray, W. M., Ostin, A., Sandberg, G., Romano, C. P. \& Estelle, M. High temperature promotes auxin-mediated hypocotyl elongation in Arabidopsis. Proc Natl Acad Sci U S A 95, 7197-7202, doi:10.1073/pnas.95.12.7197 (1998).

23 Franklin, K. A. et al. Phytochrome-interacting factor 4 (PIF4) regulates auxin biosynthesis at high temperature. Proc Natl Acad Sci U S A 108, 20231-20235, doi:10.1073/pnas.1110682108 (2011).

24 de Wit, M., Lorrain, S. \& Fankhauser, C. Auxin-mediated plant architectural changes in response to shade and high temperature. Physiol Plant 151, 13-24, doi:10.1111/ppl.12099 (2014).

25 Kim, S. et al. The epidermis coordinates thermoresponsive growth through the phyBPIF4-auxin pathway. Nat Commun 11, 1053, doi:10.1038/s41467-020-14905-w (2020).

26 Djanaguiraman, M., Boyle, D. L., Welti, R., Jagadish, S. V. K. \& Prasad, P. V. V. Decreased photosynthetic rate under high temperature in wheat is due to lipid desaturation, oxidation, acylation, and damage of organelles. BMC Plant Biol 18, 55, doi:10.1186/s12870-018-1263-z (2018).

27 Clapier, C. R., Iwasa, J., Cairns, B. R. \& Peterson, C. L. Mechanisms of action and regulation of ATP-dependent chromatin-remodelling complexes. Nature Reviews Molecular Cell Biology 18, 407, doi:10.1038/nrm.2017.26

https://www.nature.com/articles/nrm.2017.26\#supplementary-information (2017).

28 March-Díaz, R. \& Reyes, J. C. The Beauty of Being a Variant: H2A.Z and the SWR1 Complex in Plants. Molecular Plant 2, 565-577, doi:https://doi.org/10.1093/mp/ssp019 (2009).

29 Deal, R. B., Kandasamy, M. K., McKinney, E. C. \& Meagher, R. B. The Nuclear ActinRelated Protein ARP6 Is a Pleiotropic Developmental Regulator Required for the Maintenance of FLOWERING LOCUS C Expression and Repression of Flowering in Arabidopsis. The Plant Cell 17, 2633, doi:10.1105/tpc.105.035196 (2005).

30 Sura, W. et al. Dual Role of the Histone Variant H2A.Z in Transcriptional Regulation of Stress-Response Genes. The Plant Cell 29, 791, doi:10.1105/tpc.16.00573 (2017). 
31 March-Díaz, R. et al. Histone H2A.Z and homologues of components of the SWR1 complex are required to control immunity in Arabidopsis. The Plant Journal 53, 475-487, doi:10.1111/j.1365-313X.2007.03361.x (2008).

32 Jarillo, J. A. \& Piñeiro, M. H2A.Z mediates different aspects of chromatin function and modulates flowering responses in Arabidopsis. The Plant Journal 83, 96-109, doi:10.1111/tpj.12873 (2015).

33 Kazan, K. \& Lyons, R. The link between flowering time and stress tolerance. Journal of Experimental Botany 67, 47-60, doi:10.1093/jxb/erv441 (2015).

34 Holec, S. \& Berger, F. Polycomb group complexes mediate developmental transitions in plants. Plant Physiol 158, 35-43, doi:10.1104/pp.111.186445 (2012).

35 Liu, N., Fromm, M. \& Avramova, Z. H3K27me3 and H3K4me3 chromatin environment at super-induced dehydration stress memory genes of Arabidopsis thaliana. Mol Plant 7, 502-513, doi:10.1093/mp/ssu001 (2014).

36 Creyghton, M. P. et al. H2AZ is enriched at polycomb complex target genes in ES cells and is necessary for lineage commitment. Cell 135, 649-661, doi:10.1016/j.cell.2008.09.056 (2008).

37 Illingworth, R. S., Botting, C. H., Grimes, G. R., Bickmore, W. A. \& Eskeland, R. PRC1 and PRC2 are not required for targeting of H2A.Z to developmental genes in embryonic stem cells. PLoS One 7, e34848, doi:10.1371/journal.pone.0034848 (2012).

$38 \mathrm{Hu}, \mathrm{G}$. et al. H2A.Z facilitates access of active and repressive complexes to chromatin in embryonic stem cell self-renewal and differentiation. Cell Stem Cell 12, 180-192, doi:10.1016/j.stem.2012.11.003 (2013).

39 Dai, X. et al. H2A.Z Represses Gene Expression by Modulating Promoter Nucleosome Structure and Enhancer Histone Modifications in Arabidopsis. Mol Plant 10, 1274-1292, doi:10.1016/j.molp.2017.09.007 (2017).

40 Wang, Y. et al. Histone variants H2A.Z and H3.3 coordinately regulate PRC2-dependent H3K27me3 deposition and gene expression regulation in $\mathrm{mES}$ cells. BMC Biol 16, 107, doi:10.1186/s12915-018-0568-6 (2018).

41 Papamichos-Chronakis, M., Watanabe, S., Rando, O. J. \& Peterson, C. L. Global regulation of H2A.Z localization by the INO80 chromatin-remodeling enzyme is essential for genome integrity. Cell 144, 200-213, doi:10.1016/j.cell.2010.12.021 (2011).

$42 \mathrm{Xu}, \mathrm{Y}$. et al. Arabidopsis MRG domain proteins bridge two histone modifications to elevate expression of flowering genes. Nucleic Acids Research 42, 10960-10974, doi:10.1093/nar/gku781 (2014). 